\title{
JON AARONSON
}

\section{On the Pointwise Ergodic Behaviour of Transformations Preserving Infinite Measures}

Publications des séminaires de mathématiques et informatique de Rennes, 1977, fascicule 3

«Séminaire de probabilités II », , p. 1-22

$<$ http://www.numdam.org/item?id=PSMIR_1977

3_A1_0>

(C) Département de mathématiques et informatique, université de Rennes, 1977, tous droits réservés.

L'accès aux archives de la série «Publications mathématiques et informatiques de Rennes » implique l'accord avec les conditions générales d'utilisation (http://www.numdam.org/conditions). Toute utilisation commerciale ou impression systématique est constitutive d'une infraction pénale. Toute copie ou impression de ce fichier doit contenir la présente mention de copyright.

\section{NUMDAM}


ON THE POINTWISE ERGODIC BEHAVIOUR

\section{OF TRANSFORMATIONS PRESERVING INFINITE MEASURES}

Jon Aaronson

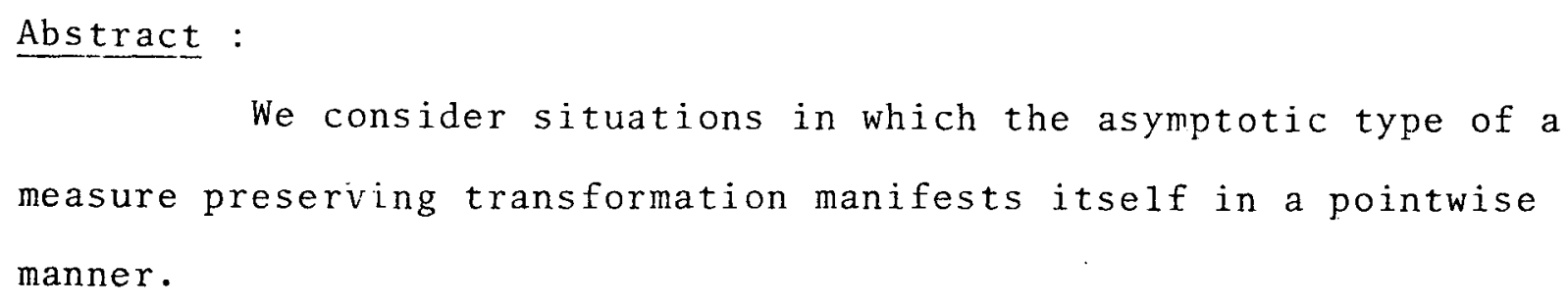

We consider situations in which the asymptotic type of a measure preserving transformation manifests itself in a pointwise manner.

\section{Abstrait :}

Nous considérons des situations où le type asymptotique d'une transformation préservant la mesure est relié à son comportement ponctuel.

Rennes, 


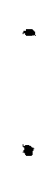


$\$ 0-$ Introduction

In $\left[2^{-}\right.$, we considered rationally ergodic measure preserving transformations, and we found that the asymptotic behaviour of such transformations depended rather heavily on their asymptotic types. Here we consider pointwise manifestations of these asymptotic types.

Let $(X, B, \mu, T)$ be a conservative measure preserving transformation. We will be considering properties of the form $\exists a_{n}>0$ s.t. $\frac{1}{a_{n}} \sum_{k=0}^{n-1} f \circ T^{k} \rightarrow \int_{X} f d \mu \quad \forall f \varepsilon L^{\prime} \quad$ in some "pointwise sense".

If $\mu(X)<\infty$, then the Birkhoff ergodic theorem states that the convergence $(0.1)$ occurs a.e. for $a_{n}=\frac{n}{\mu(X)}$. It follows from theorem 2 of [1] that if the convergence $(0.1)$ holds a.e. for some constants $a_{n}>0$, then $\mu(X)<\infty$ and $a_{n} \sim \frac{n}{\mu(x)}$. This means that the Hopf ergodic theorem $([7]$ p. 49) :

$\sum_{k=0}^{n-1} f\left(T^{k} x\right) / \sum_{k=0}^{n-1} g\left(T^{k} x\right) \rightarrow \int_{X} f d \mu / \int_{X} g d \mu \quad$ for $\mu$ a.e.x, $f, g \varepsilon L^{\prime} \int g \neq 0$ is the best possible a.e. ergodic theorem for transformations preserving infinite measures.

We wi11 investigate weaker forms of. (0.1) which are not entirely eliminated by the condition $\mu(X)=\infty$. These properties are homogeneity properties, since they will imply that $\sum_{k=0}^{n=1} p\left(T^{k} x\right)$ has uniform asymptotic behaviour on $X$ (in the sense that $\left(0.1\right.$ ) holds) for $p \in L_{+}^{1}$.

We will need to consider some weakened modes of pointwise convergence of measurable functions.

Let $\left\{f_{n}\right\}$ and $f$ be real valued measurable functions defined on $x$. We will say that $f_{n}$ converges to $f$ in $\mu$ measure $\left(f_{n} \stackrel{\mu}{\rightarrow} f\right)$ if for some, (and hence a11) finite measures $P \sim \mu$ : 


$$
\forall \varepsilon>0: P\left(\left|f_{n}-f\right| \geq \varepsilon\right) \rightarrow 0
$$

We note that $f_{n} \stackrel{\mu}{\rightarrow} f$ does not necessarily mean that $(0.2)$ holds with $\mathrm{P}=\mu$ when $\mu(\mathrm{X})=\infty$. An equivalent way of defining $\mathrm{f}_{\mathrm{n}} \stackrel{\mu}{\rightarrow} \mathrm{f}$ would be :

$$
\begin{aligned}
& \forall n_{k} \rightarrow \infty \exists m_{\ell}=n_{k_{\ell}} \rightarrow \infty \text { such that } f_{m_{\ell}} \rightarrow f \mu . a . e \text { on } x \\
& \text { We will say that } f_{n} \text { converges feebly to } f\left(f_{n}-\mu \rightarrow f\right) \text { if : }
\end{aligned}
$$

$(0,4) \quad \forall n_{k} \rightarrow \infty \exists m_{\ell}=n_{k_{\ell}} \rightarrow \infty$ such that $\frac{1}{n} \sum_{\ell=1}^{n} f_{m_{\ell}} \rightarrow f \mu \cdot a \cdot e$ on $x$.

Feeble convergence (weaker than convergence in measure) can be obtained by

$$
\begin{aligned}
& \text { Kom1os' Theorem } \\
& \text { If }[4],[9]) \\
& f_{n} \varepsilon L^{\prime}(X) \text { and } \sup _{n \geq 1} \int_{X}\left|f_{n}\right| d \mu<\infty \text { then : } \\
& f_{m_{\ell}}-m \rightarrow f
\end{aligned}
$$

In particular, if $f_{n} \rightarrow f$ weakly in $L^{\prime}$, then $f_{n} \rightarrow f$.

In $\$ 1$, we prove that if $T$ is rationally ergodic (see [2] for the definition, and [2], [3] for examples) then $\exists a_{n}>0$ such that $(0.5) \quad \frac{1}{a_{n}} \sum_{k=0}^{n-1} f \circ T^{k}-\Lambda \int_{X} f d_{\mu} \quad \forall f \varepsilon L^{\prime}$

This property, dubbed weak homogeneity, is in fact characterised by slight rational ergodicity - a very weak form of rational ergodicity :

We say that $T$ is slightly rationally ergodic if there are constants $a_{n}>0$ such that: 


$$
\forall n_{k} \rightarrow \infty \exists m_{\ell}=n_{k_{\ell}} \rightarrow \infty \text { and } A \in B, 0<\mu(A)<\infty \text {, such }
$$

that

$$
\frac{1}{a_{m_{\ell}}} \sum_{j=0}^{m_{\ell}^{-1}} \mu\left(B \cap T^{-j} C\right) \rightarrow \mu(B) \mu(C) \quad \forall B, C \in B \cap A
$$

It is evident that the sequences $\left\{a_{n}\right\}$ in $(0.6)$ and $(0.7)$ are uniquely defined up to asymptotic equality, and it will follow from theorem 1.1 that they are asymptotically equal to each other (when the same transformation $\mathrm{T}$ is involved). No ambiguity arises, therefore, if (as in [2]), we fix one such sequence $a_{n}=a_{n}(T)$, and call it a return sequence for $T$. The collection of all sequences asymptotically proportional to $a_{n}(T)$ will be called the asymptotic type of $T$ and denoted by $Q(T)$.

In the light of theorem 1.1 , we are able to refine, in $\S 3$, the results of [2] $\$ 2$ on the metric invariance of asymptotic type.

Not all c.e.m.p.t.s. are weakly homogeneous. In [6], a c.e.m.p.t. $(X, B, \mu, T)$ is constructed, which commutes with a non-singular transformation $Q: X \rightarrow X$, which does not preserve $\mu$. That is:

$$
\mathrm{QT}=\mathrm{TQ}, \mu \circ \mathrm{Q}^{-1} \sim \mu \text { and } \mu \circ \mathrm{Q}^{-1} \neq \mu
$$

In this situation, as was shown in [6], Q multiplies the measure $\mu$, and can be thought of as "squashing" $T$ into itself. We will, accordingly, call any such transformation $\mathrm{T}$ squashable. In $\$ 2$, we derive some consequences of squashability, which show that no squashable transformation is weakly homogeneous.

In $\$ 4$, we consider the stronger property of homogeneity :

(0.7) $\quad \frac{1}{a_{n}(T)} \sum_{k=0}^{n-1} f \circ T^{k} \stackrel{\mu}{\rightarrow} \int_{X} f d \mu \quad \forall f \in L^{\prime}$ 
It turns out that the homogeneity of a transformation is dependant on whether its return time stochastic processes on sets of finite measure satisfy weak laws of large numbers, and that a necessary condition for the homogeneity of a transformation, is that its asymptotic type be regularly varying with index 1 . In the case of Markov shifts, this condition is also sufficient. The methods of [1] are applicable to homogeneous transformations, and have analogous consequences.

Part of this work formed part of the author's Ph. D. thesis, (as did [1] and [2]), which was written at the Hebrew University of Jerusalem under the supervision of Professor Benjamin Weiss to whom the author is most grateful for many helpful conversations. 


\section{$\S 1$ - Weak Homogeneity}

We prove :

\section{Theorem 1.1.}

Let $(X, B, \mu, T)$ be a c.e.m.p.t. then

$\mathrm{T}$ is weakly horrogeneous iff

$T$ is sightly rationally ergodic.

(In this case, the return sequences of $T$ are asymptotically

equal)

Proof.

Assume that $T$ is weakly homogeneous. In particular assume that $T$ satisfies the convergence $(0.5)$ with the constants $a_{n}>0$ (the return sequence). We first show that

$$
\lim _{n \rightarrow \infty} \frac{1}{a_{n}} \sum_{k=0}^{n-1} \mu\left(B \cap T^{-k} C\right) \geq \mu(B) \mu(C) . \quad \forall B, C \varepsilon \mathscr{J}=\{A \varepsilon: 0<\mu(A)<\infty\}
$$

To see this, we choose $B, C \in \mathcal{F}$ and 1 et $n_{k} \rightarrow \infty$ and $\frac{1}{a_{n}} \sum_{j=0}^{n_{k}-1} \mu\left(B \cap T^{-j} C\right) \rightarrow \alpha \varepsilon[0, \infty]$

By assumption, $\exists \mathrm{m}_{\ell}=\mathrm{n}_{\mathrm{k}_{\ell}} \rightarrow \infty$ so that

$(1.2)$

$$
\frac{1}{n} \sum_{\ell=1}^{n} \frac{1}{a_{m_{\ell}}} \sum_{j=0}^{m_{\ell}-1} f \circ T^{j} \rightarrow \int_{X} f d_{\mu} \quad \text { a.e. } \forall f \varepsilon L^{\prime}
$$

We fix $f={ }^{1} \mathrm{C}$, and integrate on $B$. It follows from Fatou's lemma that $\alpha \geq \mu(B) \mu(C)$.

We now establish the slight rational ergodicity of $T$.

Let $m_{\ell} \rightarrow \infty$ be such that (1.2) is satisfied.

Fix $E \in f$, and choose (using Egorov's theorem) $A \subseteq E$ $(\mu(A)>0)$ so that the convergence $(1.2)$ for $f={ }_{E}$ is uniform on $A$. 
This implies that the convergence (1.2) for $f=1_{A}$ is bounded on A. Hence by the bounded convergence theorem :

$$
\frac{1}{n} \sum_{\ell=1}^{n} \frac{1}{a_{m_{\ell}}} \sum_{j=0}^{m_{\ell}-1} \mu\left(A \cap T^{-j} A\right) \rightarrow \mu(A)^{2}
$$

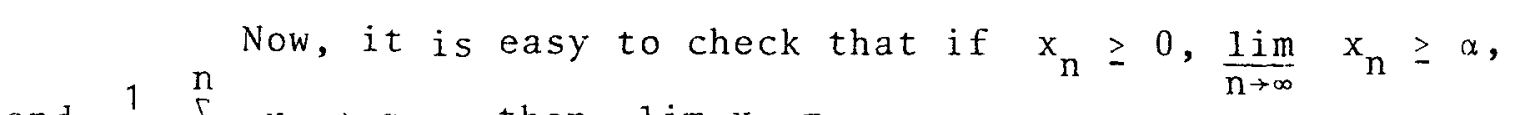
and $\frac{1}{n} \sum_{k=1}^{n} x_{k} \rightarrow \alpha$, then $\frac{\lim }{n \rightarrow \infty} x_{n}=\alpha$.

Using this, we derive from $(1.1)$ and $(1.3)$ that $\exists v_{r}={ }^{m} \ell_{r} \rightarrow \infty$ such that :

$$
\frac{1}{a_{v_{r}}} \sum_{j=0}^{\nu_{r}-1} \mu\left(A \cap T^{-j} A\right) \rightarrow \mu(A)^{2}
$$

We derive (0.6) (for $\left\{v_{r}\right\}$ ) from (1.1) and (1.4) using the method of the proof of proposition 1.1 of [2].

Now assume that $T$ is slightly rationally ergodic. In fact, assume that $\nu_{\mathrm{r}} \rightarrow \infty$ and $A \in \mathcal{f}$ satisfy $(0.6)$. In particular:

$$
\frac{1}{a_{\nu_{r}}} \sum_{j=0}^{\nu_{r}-1} 1_{A} \circ T^{k} \rightarrow \mu(A) \text { weakly in } L^{\prime}(A) \text {. }
$$

By Komlos' theorem $\exists \mathrm{m}_{\ell}={ }^{\nu} \mathrm{r}_{\ell} \rightarrow \infty$ such that

$$
\frac{1}{n} \sum_{\ell=1}^{n} \frac{1}{a_{m_{\ell}}} \sum_{j=0}^{m_{\ell}^{-1}} 1_{A} \circ T^{j} \rightarrow \mu(A) \quad \mu-\text { a.e. on } A .
$$

The set of points on which the left hand side of (1.5) converges is $T$-invariant, and, containing $A$, it must be (almost all) of $X$, by ergodicity. The Hopf ergodic theorem now establishes (1.2), and hence $(0.6)$.

Corollary 1.2 : If $\mathrm{T}$ is an invertible weakly homogeneous transformation, then so is $\mathrm{T}^{-1}$ and $\mathrm{a}_{\mathrm{n}}\left(\mathrm{T}^{-1}\right) \sim \mathrm{a}_{\mathrm{n}}(\mathrm{T})$

Proof S1ight rational ergodicity. 
The following lemma shows that no squashable transformation can be weakly homogeneous.

Lemma 2.1 :

Let $(X, B, \mu, T)$ be a squashable c.e.m.p.t. and let $m_{k} \rightarrow \infty$ and $\mathrm{d}_{\mathrm{k}}>0$. Then either :

$$
\begin{array}{lll}
\lim _{n \rightarrow \infty} \frac{1}{n} \sum_{k=1}^{n} \frac{1}{d} \sum_{k}^{m_{k}^{-1}} f \circ T^{j}=\infty & \text { a.e. } \forall f \in L_{+}^{1} \\
\text { or } \quad \frac{1}{n} \sum_{k=1}^{n} \frac{1}{d_{k}} \sum_{j=0}^{m_{k}^{-1}} f \circ T^{j} \rightarrow 0 & \text { a.e. } \forall f \in L^{1}
\end{array}
$$

Proof.

Assume $Q: X \rightarrow X, \mu \circ O^{-1} \sim \mu$ and $Q T=T Q$. We will show that if the lemma is not true, then $\mu \circ Q^{-1}=\mu$. If the conclusion to the lemma does not hold, we have, using the Hopf ergodic theorem, and the $T$-super-invariance of the $\overline{1} \overline{\mathrm{im}}$, that:

$$
\lim _{n \rightarrow \infty} \frac{1}{n} \sum_{k=1}^{n} \frac{1}{d_{k}} \sum_{j=0}^{n_{k}-1} 1_{A} \circ T^{j}=c \mu(A) \text { a.e. } \forall A \varepsilon B \text { where } 0<c<\infty
$$

Now since $\mu \circ \mathrm{Q}^{-1} \sim \mu$, we have that

$$
\lim _{n \rightarrow \infty} \frac{1}{n} \frac{1}{d_{k}} \sum_{j=0}^{n_{k}-1} 1_{A} \circ T^{j} \circ Q=c \mu(A) \text { a.e. } \quad \forall A \in B
$$

And since $Q T_{\mathrm{n}_{\mathrm{k}}}=\mathrm{TQ}$, we have that the left hand side of $(2.2)$ is $\varlimsup_{n \rightarrow \infty} \frac{1}{n} \sum_{k=1}^{n} \frac{1}{d_{k}} \sum_{j=0}^{k} 1 Q^{-1}{ }_{A}^{\circ} T^{j}$. Hence, by (3.1) and (3.2), we have $\mu\left(Q^{-1} A\right)=\mu(A) \quad \forall A \in G$

\section{Theorem 2.2}

Let $(X, B, \mu, T)$ be a squashable c.e.m.p.t. and let $A, B \in B \quad 0<\mu(A), \mu(B)<\infty \quad a_{n}=\sum_{k=0}^{n-1} \mu\left(A \cap T^{-k} B\right)$. Then : 


$$
\frac{1}{a_{n}} \sum_{k=0}^{n-1} f \circ T^{k} \stackrel{\mu}{\longrightarrow} \cup \quad \forall f \in L^{1}
$$

Proof.

$$
\begin{aligned}
& \text { Let } n_{k} \rightarrow \infty: \\
& \int_{A} \frac{1}{a_{n_{k}}} \sum_{j=0}^{n_{k}-1} 1_{B} \circ T^{j} d_{\mu}=1 \quad \forall k \geq 1
\end{aligned}
$$

By Komlos' theorem, $\exists \emptyset \varepsilon L^{1}(A)$ and $m_{\ell}=n_{k_{\ell}} \rightarrow \infty$ such that

$$
\frac{1}{n} \sum_{\ell=1}^{n} \frac{1}{a_{m}} \sum_{j=0}^{m_{\ell}-1} 1_{B} \circ T^{j} \rightarrow \emptyset \quad \text { a.e. on } A \text {. }
$$

The set on which this convergence occurs is T-invariant, as is the limit function. Therefore, the convergence (2.3) takes place a.e. on $X$ and the limit function is constant. The constant must be finite since it is integrable on $A$. But $T$ is squashable, so by lemma 3.1 , the only possible value for this constant is 0 .

$$
\text { We now choose a subset } C \text { of } A(\mu(C)>0) \text { on which the }
$$

convergence (2.3) is uniform. Integrating, we obtain:

$$
\frac{1}{n} \sum_{\ell=1}^{n} \frac{1}{a_{m_{\ell}}} \sum_{j=0}^{m_{\ell}-1} \mu\left(C \cap T^{-j} B\right) \rightarrow 0
$$

This implies that $\exists \nu_{r}=m_{\ell_{r}} \rightarrow \infty$ such that

$$
\frac{1}{a_{v_{r}}} \sum_{j=0}^{v_{r}^{-1}} \mu\left(\mathrm{C} \cap \mathrm{T}^{-j_{B}}\right) \rightarrow 0
$$

From here; we see that $\exists p_{n}={ }^{\nu} r_{n} \rightarrow \infty$ such that

$$
\frac{1}{a_{p_{n}}} \sum_{j=0}^{p_{n}^{-1}} 1_{B} \circ T^{j} \rightarrow 0 \text { a.e. on } C \text {. }
$$

As before, this convergence must take place a.e. on $X$, and using the Hopf ergodic theorem, we have 


$$
\frac{1}{a_{p_{n}}} \sum_{j=0}^{p_{n}^{-1}} f \circ T^{j} \rightarrow 0 \quad \text { a.e. } \forall f \in L^{1}
$$

\section{$\S 3$ - Metric invariance of asymptotic type}

In this section, we deduce the invariance of the asymptotic. type of weakly homogeneous transformations under the metric equivalences defined in [2] $\$ 2$. We will also obtain that homogeneity properties are invariant under these equivalences, whose definitions we now recall.

Let $\left(X_{i}, B_{i}, \mu_{i}, T_{i}\right)$ be m.p.t.s., and let $0<c<\infty$. We will say :

$$
\pi: X_{1} \rightarrow X_{2}, \pi^{-1} B_{2} \subseteq 1, \pi_{1}=T_{2} \pi \text { and } \mu_{1} \circ \pi^{-1}=c \mu_{2}
$$

that $T_{2}$ is a factor of $T_{1}\left(T_{1} \rightarrow T_{2}\right)$ if there is a c-map of $\mathrm{T}_{1}$ onto $\mathrm{T}_{2}$ for some $0<\mathrm{c}<\infty$

and that $\mathrm{T}_{1}$ is similar to $\mathrm{T}_{2}\left(\mathrm{~T}_{1} \sim \mathrm{T}_{2}\right)$ if $\mathrm{T}_{1}$ and $\mathrm{T}_{2}$ are both factors of the same m.p.t.

The following is immediate from the definitions:

\section{Proposition 3.1 .}

Let $\mathrm{T}_{1}, \mathrm{~T}_{2}$ be c.e.m.p.t.s. and let $\pi: \mathrm{T}_{1} \stackrel{\mathrm{c}}{\rightarrow} \mathrm{T}_{2}$. Then:

$\mathrm{T}_{1}$ is homogeneous (weakly homogeneous) $\Leftrightarrow$

$\mathrm{T}_{2}$ is homogeneous (weakly homogeneous), and in either case :

$$
\frac{a_{n}\left(T_{2}\right)}{a_{n}\left(T_{1}\right)} \rightarrow c \quad \text { as } n \rightarrow \infty
$$




\section{Theorem 3.2}

Let $\mathrm{T}_{1}, \mathrm{~T}_{2}$ be c.e.m.p.t.s., and let $\mathrm{T}_{1} \sim \mathrm{T}_{2}$. Then:

$\mathrm{T}_{1}$ is homogeneous (weakly homogeneous) iff

$\mathrm{T}_{2}$ is homogeneous (weakly homogeneous) and in either case $Q\left(\mathrm{~T}_{1}\right)=Q\left(\mathrm{~T}_{2}\right)$.

proof.

We will show that if $\mathrm{T}_{1}$ is homogeneous, then so is $\mathrm{T}_{2}$ and $Q\left(T_{1}\right)=Q\left(T_{2}\right)$. The proof for weak homogeneity is analogous. So we assume that :

$$
\frac{1}{a_{n_{k}}\left(T_{1}\right)} \sum_{j=0}^{n_{k}-1} p \circ T_{1}^{j} \rightarrow \int_{X} p d \mu, \mu_{1}-a \cdot e . \quad \forall p \in L^{1}\left(X_{1}\right)
$$

and that $\pi_{1}: \mathrm{S} \stackrel{\mathrm{C}}{\rightarrow} \mathrm{T}_{1}, \pi_{2}: \mathrm{S} \stackrel{\mathrm{d}}{\rightarrow} \mathrm{T}_{1}$ where $(Y, f, v, S)$ is a m.p.t. necessarily conservative since $T_{1}$ is conservative.

The Hopf ergodic theorem applied to $S$ states that

$$
\sum_{k=0}^{n-1} f\left(S^{k} x\right) / \sum_{k=0}^{n-1} g\left(S^{k} x\right) \rightarrow h \text { v.a.e. } \forall f, g \in L^{1}(Y), g>0 \text { a.e. }
$$

where $h \circ S=h, \int_{Y}|h g| d v<\infty$ and $\int_{Y} h g d v=\int_{Y} f d v$

Combining (3.1) with (3.2) and the fact that $\mathrm{T}_{1}$ is a c-factor of S yields that

$$
\frac{1}{a_{n_{k}}\left(T_{1}\right)} \sum_{j=0}^{n_{k}-1} f \circ S^{j} \rightarrow h \quad \text { v.a.e. } \quad \forall f \in L^{\prime}(Y)
$$

where $h \circ S=h$ and $\forall p \in L^{1}\left(X_{1}\right): h \cdot p \circ \pi_{1} \in I^{1}(Y)$ and

$$
\int_{Y} h p \circ \pi_{1} d v=\int_{X_{1}} p d \mu_{1} \int_{Y} f d v
$$

In particular, if $h$ is constant, then

$$
h=\frac{1}{c} \int_{Y} f d v
$$


Now choose $q \varepsilon L^{1}\left(x_{2}\right)$. We have from (3.3) that

$$
\frac{1}{a_{n_{k}}\left(T_{1}\right)} \sum_{j=0}^{n_{k}-1} q \circ T_{2}^{j} \circ \pi_{2}=\frac{1}{a_{n_{k}}(T)} \sum_{j=0}^{n_{k}-1} q \circ \pi_{2} \circ S^{j} \rightarrow h \quad v-a \cdot e .
$$

where $h \in \dot{L}^{\infty}, h \circ S=h$. The sequence of functions on the left hand side of (3.5) are all $\pi_{2}^{-1} \mathrm{~B}_{2}$ - measurable, and hence, so is $h$. This means that there is a $k: X_{2} \rightarrow R$ such that $h=k 0 \pi_{2}$

Thus $k$ is a constant, and so is h. By (3.4):

$$
h=\frac{1}{c} \int_{X} q \circ \pi_{2} d \nu=\frac{d}{c} \int_{X}{ }_{2} q^{d} \mu_{2}
$$

The fact that $\nu \circ \pi_{2}^{-1}=d \nu_{2}$ implies that

$$
\frac{1}{a_{n_{k}}\left(T_{1}\right)} \sum_{j=0}^{n_{k}-1} f \circ T_{2}^{j} \rightarrow \frac{d}{c} \int_{X_{2}} f d \mu_{2} \quad \mu_{2}-a \cdot e \cdot \quad \forall f \in L^{1}\left(X_{2}\right)
$$

\section{$\$ 4$ - Homogeneity}

In this section, we consider homogeneity. The homogeneity of a transformation is dependant on whether its return time stochastic processes on sets of finite measure satisfy weak laws of large numbers.

By a stochastic process, we mean a quintuple $(\Omega, Q, P, \sigma, \phi)$ where $(\Omega, a, P, \sigma)$ is a m.p.t.,$P(\Omega)=1$ and $\phi$ is a real valued measureable function. In this section, we will on $1 y$ be considering ergodic N-valued stochastic processes (i.e. where $\sigma$ is ergodic and $\phi: \Omega \rightarrow N$ ). We will say that an stochastic process $(\Omega, Q, P, \sigma, \phi)$ has a weak law of large numbers (W.L.L.N.) if there are constants $b_{n}>0$ such that: 
(4.1) $\quad \frac{1}{b_{n}} \sum_{k=0}^{n \sim 1} \phi \circ \sigma^{k} \stackrel{P}{\rightarrow} 1$

Now let $(x, \delta, \mu, T)$ be a c.e.m.p.t. and let $\Omega \in B$ $0<\mu(\Omega)<\infty$. We define the return time stochastic process of $\mathrm{T}$ on $\Omega$; $(\Omega Q, P, \sigma, \phi)$ by

$$
\begin{aligned}
& Q=B \cap \Omega, \quad P(A)=\mu(A \cap \Omega) / \mu(\Omega), \\
& \left.\phi(x)=\inf \{n \geq 1\}: T^{n} x \in \Omega\right\}- \text { the return time function } \\
& \sigma x=T^{\phi(x)} \times\left(=T_{\Omega} x-\text { the induced transformation }\right) .
\end{aligned}
$$

It follows ([8]) that $\sigma$ is ergodic and that any (N-valued, ergodic) stochastic process is the return time stochastic process of a c.e.m.p.t. The formula of Kac states that $\int_{\Omega} \phi d P=\mu(X)$. In case this quantity is finite, then by Birkhoff's ergodic theorem, $T$ is homogeneous and $(\Omega, a, P, \sigma, \phi)$ satisfies a strong law of large numbers. The main result of this section is :

\section{Theorem 4.1}

Let $(X, G, \mu, T)$ be a c.e.m.p.t., $\Omega \in \mathcal{f}$ and $(\Omega . Q, P, \sigma, \phi)$ be the return time stochastic process of $T$ on $\Omega$.

Then : $T$ is homogeneous (with return sequence $\left\{a_{n}\right\}$ ) iff $(\Omega, a, P, \sigma, \phi)$ satisfies a W.L.L.No (with constants $\left.\left\{b_{n}\right\}\right)$. In this case, $\left\{a_{n}\right\}$ and $\left\{b_{n}\right\}$ are regularly variying with index 1 , and

(4.2) $\quad \mu(\Omega) a\left[b_{n}\right]^{\sim} \frac{1}{\mu(\Omega)} \quad b\left[a_{n}\right]^{\sim} n$ as $n \rightarrow \infty$

A function $a: \mathbb{R}_{+} \rightarrow \mathbb{R}_{+}$is termed regularly varying with index $\underline{\alpha \in \mathbb{R}}$ if

$$
\frac{a(\lambda t)}{a(t)} \underset{t \rightarrow \infty}{\longrightarrow} \lambda^{\alpha} \quad \forall \lambda>0
$$


A sequence $\left\{a_{n}\right\}$ is so termed if the function $a[t]$ has this property (where $[t]$ denotes the largest integer not greater than $t$ ). Regular variation is discussed in [5] and [10].

The proof of theorem 4.1 will be in a sequence of lemmas. Firstly, let, for $x \in \Omega$ :

$$
\psi_{n}(x)=\sum_{k=1}^{n} 1_{\Omega}\left(T^{k} x\right) \text { and } \phi_{n}(x)=\sum_{k=0}^{n-1} \phi\left(\sigma^{k} x\right)
$$

It follows that :

$(4.3)$

$$
\psi_{\phi_{n}}(x)(x)=n \text { and } \psi_{\phi_{n}}(x)(x) \leq n<\phi_{\psi_{n}}(x)+1(x)
$$

We will assume that $\mu(\Omega)=1$. No generality is lost, because we can normalise the measure $\mu$, and (4.2) will follow from the regular variations of $a_{n}$ and $b_{n}$.

Lemma 4.2

$T$ is homogeneous with return sequence $\left\{a_{n}\right\}$ iff

Proof.

$$
\frac{\psi_{n}}{a_{n}} \stackrel{p}{\rightarrow} 1
$$

If $T$ is homogeneous, then (4.4) is evident. Conversely, we have that $\forall \mathrm{n}_{\mathrm{k}} \rightarrow \infty \exists \mathrm{m}_{\ell}=\mathrm{n}_{\mathrm{k}_{\ell}} \rightarrow \infty$ such that

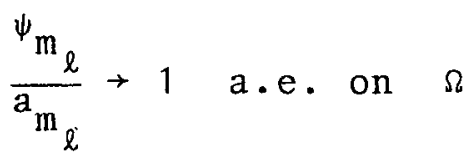

The convergence set for $(4.5)$ is T-invariant, and so, containing $\Omega$, must be (almost all of) $X$. Homogeneity now follows from the Hopf ergodic theorem.

\section{Lemma 4.3}

Let $a: R_{+} \rightarrow R_{+}$be monotone. If $V \varepsilon>0, m \in N:$ 


\section{$(4.6)$}

$$
a((1-\varepsilon) m t) \leq m a(t) \leq a((1+\varepsilon) m t) \text { for } t \text { large then } a(t) \text { is }
$$

regularly varying with index 1 .

\section{Proof.}

A little manipulation shows that (4.6) is true for a positive rational number. Now suppose that $\lambda, \varepsilon$ are positive rational numbers. We have:

$$
\lambda a(t)=(1+\varepsilon)\left(\frac{\lambda}{1+\varepsilon}\right) a(t) \leq(1+\varepsilon) a\left((1+\varepsilon)\left(\frac{\lambda}{1+\varepsilon}\right) t\right)=(1+\varepsilon) a(\lambda t)
$$

for $t$ large and similarly that

$$
\lambda a(t) \geq(1-\varepsilon) a(\lambda t) \text { for } t \text { large. }
$$

In other words :

$$
\frac{a(\lambda t)}{a(t)} \underset{t \rightarrow \infty}{\longrightarrow} \lambda \text { for } \lambda>0, \lambda \varepsilon Q \text {. }
$$

The required regular variation of a(t) now follows from its momtonicity.

\section{Lemma 4.4}

Let $b_{n} \sim b_{n+1} \rightarrow \infty$ and $b_{m n} \sim m b_{n}$ as $n \rightarrow \infty \quad \forall m \in \mathbb{N}$. Then $a(t)=\min \left\{n \geq 1: b_{n} \geq t\right\}$ is regularly varying with index 1 , and $a_{b(t)} \sim t$ as $t \rightarrow \infty$

Proof.

We will show (4.6). It follows easily from the assumptions that :

(i) $b_{\left[\frac{n}{m}\right]}^{2} \frac{1}{m} b_{n}$ as $n \rightarrow \infty \quad \forall m \in N$

(ii) $b_{a(t)} \geq t>b_{a(t)-1} \quad \forall t \geq 0$ where $b_{0}=0$

(iii) $b_{a(t)} \sim t$ as $t \rightarrow \infty$ 
Using these, we can see that $\forall \varepsilon>0, m \varepsilon N, \exists t_{0}$ such that $\forall t \geq t_{0}:$

$$
\mathrm{b}_{\mathrm{ma}(t)} \geq(1-\varepsilon) \mathrm{mb} a(t) \geq(1-\varepsilon) \mathrm{mt} \text {. }
$$

and

$$
b^{b}\left[\frac{a((1+\varepsilon) m t)}{m}\right] \geq \frac{1}{(1+\varepsilon) m} b_{a((1+\varepsilon) m t) \geq t} \text {. }
$$

These inequalities yield (4.6) immediately.

\section{Lemma 4.5}

If $\frac{\phi_{n}}{b_{n}} \stackrel{p}{\rightarrow} 1$ then $T$ is homogeneous with return sequence $a(n)$, where $a(t)=\min \left\{n \geq 1: b_{n} \geq t\right\}$ is regularly varying with index 1 , and $b_{a(t)} \sim t$ as $t \rightarrow \infty$

Proof.

We first establish the regular variation of $a(t)$, and the fact that $b_{a(t)} \sim t$ as $t \rightarrow \infty$. To do this, in view of the preceding lemma, it is sufficient to show that $b_{n} \sim b_{n+1}$ and $b_{m n} \sim m b_{n}$ as $\mathrm{n} \rightarrow \infty \forall \mathrm{m} \in \mathbb{N}$. The first of these is clear. To see the second, let $\varepsilon>0$ and $m \varepsilon \mathbb{N}$. Since

$$
\underset{\mathrm{b}_{\mathrm{n}}}{\stackrel{\mathrm{p}}{\rightarrow}} \stackrel{\mathrm{p}}{\rightarrow}
$$

there is an $n_{0}$ such that $\forall n \geq n_{0}$ :

$$
P\left(\prod_{k=0}^{m-1} \sigma^{-k n}\left[\left|\frac{\phi n}{b_{n}}-1\right| \leq \varepsilon\right] \cap\left[\left|\frac{m n}{b_{m n}}-1\right| \leq \dot{\varepsilon}\right]>0\right.
$$

That is: $\forall \mathrm{n} \geq \mathrm{n}_{0} \exists \times \varepsilon \Omega \quad$ such that

$$
(1-\varepsilon) b_{n} \leq \phi_{n}\left(\sigma^{k n} x\right) \leq(1+\varepsilon) b_{n} \text { for } 0 \leq k \leq m-1
$$

and

$$
(1-\varepsilon) b_{m n} \leq \phi_{m n}(x) \leq(1+\varepsilon) b_{m n}
$$

These, together with the fact that

$$
\phi_{m n}(x)=\sum_{k=0}^{m-1} \phi_{n}\left(\sigma^{k n} x\right)
$$


show that

$$
\left|\frac{b_{m n}}{b_{n}}-m\right| \leq \frac{2 \varepsilon}{1-\varepsilon} \quad \text { for } \quad n \geq n_{0}
$$

To complete the proof of this lemma, it is now sufficient to show that:

$$
\text { (4.7) } \forall \varepsilon>0 \quad P\left(a((1-\varepsilon) n) \leq \psi_{n} \leq a((1+\varepsilon) n)\right) \rightarrow 1
$$

(This, because of the now established regular variation of $a(t)$, and lemma 4.2$)$

$$
\begin{aligned}
& \text { In view of }(4.3) \text {, we have: } \\
& \psi_{n} \geq a((1+\varepsilon) n) \Rightarrow \phi_{a}((1+\varepsilon) n) \leq n \leq \frac{1}{1+\varepsilon} b_{a}((1+\varepsilon) n) \text {, and } \\
& \psi_{n}<a((1-\varepsilon) n) \Rightarrow \phi_{a}((1-\varepsilon) n) \geq n \geq \frac{1}{1-\varepsilon} b_{a}((1-\varepsilon) n) \\
& \text { The assumption } \phi_{\frac{n}{n}} \rightarrow 1 \text { thus establishes (4.7) }
\end{aligned}
$$

Lemma 4.6

Suppose $T$ is homogeneous with return sequence $\left\{a_{n}\right\}$. Then $b(t)=\inf \left\{n \geq 1: a_{n} \geq t\right\}$ is regularly varying with index $1, a_{b(t)} \sim t$ as $t \rightarrow \infty$, and

$$
\frac{\phi_{n}}{b(n)} \stackrel{p}{\rightarrow} 1
$$

Proof.

It is evident that :

$$
\begin{aligned}
& \text { (i) } a_{n} \sim a_{n+1} \text { as } n \rightarrow \infty \\
& \text { (ii) } a_{b(t)} \geq t>a_{b(t)-1} \text { for } t \geq 0 \text { where } a_{0}=0 \text {, and : } \\
& \text { (iii) } a_{b(t)} \sim t \text { as } t+\infty
\end{aligned}
$$

Thus, from (4.3), we have, $\forall \varepsilon>0$ that for $t$ large :

$$
\phi[t]>b((1+\varepsilon) t) \rightarrow \psi_{b}((1+\varepsilon) t) \leq[t] \leq \frac{1}{1+\varepsilon} a_{b}((1+\varepsilon) t)
$$




$$
\phi[t] \leq b((1-\varepsilon) t) \Rightarrow \psi_{b(1-\varepsilon) t)} \geq[t] \geq \frac{1-\varepsilon / 2}{1-\varepsilon} a_{b}((1-\varepsilon) t)
$$

Whence, by lemma $4.2, \forall \varepsilon>0$ :

$$
P(b((1-\varepsilon) t) \leq \phi[t] \leq b((1+\varepsilon) t)) \underset{t \rightarrow \infty}{\rightarrow} 1
$$

To prove the lemma, it is sufficient now to show the regular variation of $b(t)$. As in the proof of 1 emma 4.5 , we deduce from that $\forall \varepsilon>0 m \varepsilon N \exists t_{0}$ such that $V t \geq t_{0} \exists x \varepsilon \Omega$ such that :

$$
b((1-\varepsilon) t) \leq \phi[t]\left(\sigma^{k[t]} x\right) \leq b((1+\varepsilon) t) \text { for } 0 \leq k \leq m-1
$$

and

$$
b((1-\varepsilon) m t) \leq \phi_{m[t]}(x) \leq b((1+\varepsilon) m t)
$$

Since $\quad \phi_{m[t]}(x)=\sum_{k=0}^{m-1} \phi_{[t]}\left(\sigma^{k[t]} x\right)$, we have established $(4.6)$ and hence, the regular variation of $b(t)$.

Lemmas 4.5 and 4.6 constitute the proof of theorem 4.1 , as, the regular variations of the sequences $\left\{a_{n}\right\}$ and $\left\{b_{n}\right\}$ of lemmas 4.6 and 4.5 respectively, are established by 1 emmas 4.5 and 4.6 respectively.

We note that if $T$ is both homogeneous, and weakly rationally ergodic (see[2]) then $T$ satisfies a "strong $\mathrm{L}^{1}$ ergodic theorem" on certain sets. Namely, if $A \in R(T)$ and $B \leqq A$ then

$$
\int_{A}\left|\frac{1}{a_{n}(T)} \sum_{k=0}^{n-1} 1_{B} \circ T^{k}-\mu(B)\right| d \mu \rightarrow 0 \text { as } n \rightarrow \infty
$$

This is because

$$
\frac{1}{a_{n}(T)} \sum_{k=0}^{n-1} 1_{B} \circ T^{k} \rightarrow \mu(B)
$$

both in measure (by homogeneity) and weakly in $\mathrm{L}^{1}(\mathrm{~A})$ (by weak rational ergodicity) and hence : strongly in $\mathrm{L}^{1}(\mathrm{~A})$. 


\section{Theorème 4.2}

If $(X, B, \mu, T)$ is a homogeneous m.p.t. and $\mu(X)=\infty$ then: $\frac{1 \mathrm{im}}{\mathrm{n} \rightarrow \infty} \frac{1}{\mathrm{a}_{\mathrm{n}}(\mathrm{T})} \sum_{k=0}^{n-1} f \circ T^{k}=0$ a:e. $\forall f \in L^{1}+$

Proof. (This is an adaptation of the proof of theorem 2 of [1]).

Suppose otherwise, then $\exists \alpha>0 \quad(\alpha \leq 1)$ such that

$$
\frac{\lim }{n \rightarrow \infty} \frac{1}{a(n)} \sum_{k=0}^{n-1} 1_{A} \circ T^{k}=\alpha \mu(A) \text { a.e. } \quad \forall A \in B \quad\left(a(n)=a_{n}(T)\right) \text {. }
$$

Fix $A \in B, \mu(A)=2$. Then $\exists \Omega \leq A, \mu(\Omega)=1$ such that

$$
\frac{1}{a(n)} \sum_{k=0}^{n-1} 1 A\left(T^{k} x\right) \geq \delta>0 \quad \forall x \in \Omega, n \geq 1
$$

We now restrict attention to $(\Omega, Q, p, \sigma, \phi)$-the return time stochastic process of $\mathrm{T}$ on $\Omega$. We will show $\int_{\Omega} \phi \mathrm{dP}<\infty$, using wherewherever possible, the proof of theorem 2 of [1].

$$
\text { Let } c(t)=P(\phi \geq t) \text { and } L(t)=\int_{0}^{t} c(s) d s \text {. }
$$

We have by theorem 4.1 that

$$
\underset{b\left(\frac{n}{n}\right)}{\stackrel{p}{\rightarrow}} 1 \text {, where } b(n) \text { is regularly varying with index } 1
$$

and $b(a(n)) \sim a(b(n)) \sim n$ as $n \rightarrow \infty$.

By step 2 to the proof of theorem 2 of [1] , we have

$$
\begin{aligned}
& \sum_{n=1}^{\infty} c(b(n))<\infty \\
& \text { Let } \hat{b}(n)=\sum_{k=0}^{n-1} L(b(k)) . \text { If we show that }
\end{aligned}
$$

(4.11) $\quad \frac{1 i m}{n \rightarrow \infty} \frac{\hat{b}(n)}{b(n)} \geq 1$

Then (in view of the regular variation of $b(n)$ ), we have by step 4 of the above mentioned proof, that 
$\sum_{n=1}^{\infty} c \hat{b}(n)<\infty$

and hence that $\int_{\Omega} \phi d P<\infty$. We establish (4.11):

Let $f_{n}(x)=\left\{\begin{array}{crl}\phi(x) & \text { if } & \phi(x) \leq b(n) \\ 0 & \text { else }\end{array}\right.$

By (4.10), we have that for P.a.e. $x \varepsilon \Omega$

$\phi\left(\sigma^{n} x\right) \neq f_{n}\left(\sigma^{n} x\right)$ for only finitely many $n \geq 1$.

In particular :

$\frac{1}{\mathrm{~b}(\mathrm{n})} \sum_{\mathrm{k}=0}^{\mathrm{n}} \mathrm{f}_{\mathrm{k}} \circ \sigma^{\mathrm{k}} \stackrel{\mathrm{p}}{\rightarrow} 1$

Choosing a.e.-convergent subsequences, and using Fatou's

lemma, we see that

$\frac{\lim }{\mathrm{n} \rightarrow \infty} \frac{1}{\mathrm{~b}(\mathrm{n})} \sum_{\mathrm{k}=0}^{\mathrm{n}-1} \int_{\Omega} \mathrm{f}_{\mathrm{k}} \mathrm{dP} \geq 1$

This establishes (4.11) because

$\int_{\Omega} f_{k} d P \leq L(b(k))$

Corollary : If $(\Omega, a, P, \sigma, \phi)$ is an (ergodic, $N$-valued) stochastic process, and $\frac{\phi_{n}}{b_{n}} \stackrel{p}{\rightarrow} 1$ then

$$
\begin{aligned}
& E(\phi)=\infty \Rightarrow \overline{\operatorname{Iim}}_{n \rightarrow \infty} \frac{\phi_{n}}{\hbar_{n}}=\infty \text { a.e. } \\
& \text { It follows from the remarks following corol- }
\end{aligned}
$$

lary 3.5 of [3] that no odd restriction (of an inner function of $\mathbf{R}^{2+}$ ) preserving an infinite measure can be homogeneous.

The rest of this section is devoted to classifying the homogeneous Markov shifts. The following theorem of $W$. Feller will be used:

Theorem $4.3([5]$ p.236)

Let $\left\{X_{n}\right\}_{n=1}^{\infty}$ be independent, identically distributed random variables (i.i.d.r.v.s) with $x_{k} \geq 0$.

Let $c(t)=P\left(X_{1} \geq t\right)$ and $L(t)=\int_{0}^{t} c(s) d s$. 
Then :

$\exists \quad b(n)$ such that $\frac{1}{b(n)} \sum_{k=1}^{n} x_{k} \rightarrow 1$ in probability iff $L(t)$ is slowly varying (i.e. regularly varying with index 0 ) and in this case :

$b(n) \sim n L(b(n))$ as $n \rightarrow \infty$

Let $(X, B, \mu, T)$ be a c.e.m.p.t.

We recal1 from [2], that a set $\Omega \in \&$ was said to be a recurrent event for $T$ if $\forall 0=n_{0} \leq n_{1} \leq n_{2} \leq \cdots \leq n_{k}$ :

$$
\mu\left(\prod_{j=1}^{k} T^{-n_{j}} \mathrm{j}_{\Omega}=\mu(\Omega) \prod_{j=1}^{k} u_{n j}-n_{j-1} \quad\right. \text { where }
$$

$u_{n}=\mu\left(\Omega \cap T^{-n_{\Omega}}\right) / \mu(\Omega) \quad$.

The collection of recurrent events for $T$ is denoted by $M(T)$, . and $T$ is said to admit recurrent events if $M(T) \neq \emptyset$. It is not hard to see that $M(T) \neq \emptyset$ iff $T$ has a Markov shift factor, and that if $(\Omega, a, P, \sigma, \phi)$ is the return time stochastic process of $T$ on $\Omega \varepsilon M(T)$ then

$$
\left\{\begin{array}{lll}
\phi & \circ & \sigma^{\mathrm{n}}
\end{array}\right\} \text { are i.i.d.r.v.s and }
$$

$$
\sum_{n=0}^{\infty} u_{n} \lambda^{n}=\left((1-\lambda) \sum_{n=0}^{\infty} c_{n} \lambda^{n}\right)^{-1}
$$

where $\quad u_{n}=\frac{\mu\left(\Omega \cap T^{-n} \Omega\right)}{\mu(\Omega)}$ and $c_{n}=P(\phi>n)$

lt was shown in [2] that if $\exists \Omega \varepsilon M(T)$ then $T$ is rationnally ergodic (hence by theorem 1.1 weakly homogeneous), and that $\frac{1}{\mu(\Omega)} \sum_{k=0}^{n} u_{k}$ is a return sequence for $T$.

Now suppose that $T$ is a c.e.m.p.t. and that $\Omega \in M(T)$, $\mu(\Omega)=1$. The equation (4.12) implies, through Karamata's Tauberian theorem (see [5] and [10]) that 


$$
\begin{aligned}
& \sum_{k=0}^{n} u_{k} \text { is regularly varying with index } 1 \text { iff } \\
L(n)= & \sum_{k=0}^{n} c_{k} \text { is slowly varying. }
\end{aligned}
$$

Thus, if $\mathrm{T}$ is homogeneous, then $\mathrm{L}(\mathrm{n})$ is slowly varying; and, by Feller's theorem, if $L(n)$ is slowly varying, then $(\Omega, \hat{i}, \mathrm{P}, \sigma, \phi)$ satisfies a W.L.L.N.

We have proved

\section{Theorem 4.4}

If $T$ is a c.e.m.p.t. and $M(T) \neq \emptyset$, then $T$ is homogeneous iff $a_{n}(T)$ is regularly varying with index 1 .

\section{$\underline{\text { References }}$}

[1] J. Aaronson : On the ergodic theory of non-integrable functions and infinite measure spaces. Israel Journal of Mathematics 27, $\mathrm{n}^{\circ} 2$ (1977) pp 163-173

[2] J. Aaronson : Rational ergodicity and a metric invariant for Markov shifts. Ibid. pp 93-123

[3] J. Aaronson : Ergodic theory for inner functions of the upper half plane. Submitted to Ann. I.H.P.

[4] S.D. Chatterji : A general strong law. Inventiones Maths. 9 (1970) pp 235-245.

[5] W. Feller : Introduction to Probability Theory and its applications. vo1. 2 Wi-ley, N.Y. (1966)

[6] A. Hajian, Y. Ito and S. Kakutani : Invariant measures and orbits of dissipative transformations. Advances in Math. $\underline{9}$ (1972) pp $52-66$

[7] E. Hopf : Ergodentheorie : Chelsea N.Y.

[8] S. Kakutani : Induced measure preserving transformations, Proc. Imp. Acad. Sci. Tokyo 19 (1943) pp 635-641

[9] J: Komios : A generalisation of a problem of Steinhaus. Acta. Math. Acad. Sci. Hung. 18 (1967) pp 217-229 
[10] E. Seneta : Regularly varying functions : Springer Lecture notes $\underline{508}$, Berlin (1976) 